\title{
Association of Aldosterone, Plasma Renin Activity (PRA) and Superoxide Dismutase (SOD) with Inflammation and Insulin Resistance in Adult Men with Central Obesity
}

\author{
Hera Yuliana Intantri ${ }^{1,2^{*}}$, Andi Wijaya ${ }^{1,2}$, Ilhamjaya Patellongi ${ }^{2}$
}

${ }^{1}$ Prodia ChildLab, Jl. Kramat Raya 53, Jakarta, Indonesia

${ }^{2}$ Postgraduate Program, Hasanuddin University, Jl. Perintis Kemerdekaan Km 10, Makassar, Indonesia ${ }^{\star}$ Correspondence: hera_yuliana@yahoo.com

\section{Abstract}

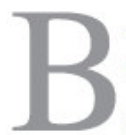

ACKGROUND: Visceral Obesity is related with chronic low grade inflammation, and is the main component of metabolic syndrome (MetS). MetS is associated with increased cardiovascular disease (CVD). Furthermore, superoxide dismutase (SOD) is correlated with insulin resistance. Several studies have reported a strong correlation between Renin Angiotensin Aldosterone System (RAAS) and CVD, but the association of Aldosterone, Plasma Renin Activity (PRA) and SOD with inflammation, insulin resistance and MetS have not been fully elucidated. The aim of this study was to investigate the correlation of Aldosterone, PRA, and SOD with inflammation (high sensitivity c-reactive protein/hsCRP) and insulin resistance (homeostasis model assessment-insulin resistance/HOMA-IR) in adult men with central obesity.

METHODS: This was a cross-sectional study, which was carried out on 80 male subjects with central obesity who were divided into 2 groups: the group of subjects who had fulfilled the MetS criteria and the other group of subjects who did not. After an overnight fasting, blood pressure (BP) was measured on all subjects and laboratory examinations were done for measurement of the concentration of fasting glucose, high density lipoprotein cholesterol (HDL-C), triglyceride, hsCRP, insulin, aldosterone, PRA, and SOD.

RESULTS: We found aldosterone had positive correlation with PRA $(\mathrm{r}=0.389 ; \mathrm{p}<0.001)$ and triglycerides $(\mathrm{r}=$ $0.234 ; \mathrm{p}=0.036)$. PRA had positive correlation with SOD $(r=0.220 ; p=0.05)$ and HDL-C $(r=0.273 ; p=0.014)$, but not with hsCRP $(r=-0.044 ; \mathrm{p}=0.696)$ and HOMAIR $(r=0.168 p=0.136)$. PRA correlated with HOMAIR in MetS $(r=0.471 ; p=0.01)$. Aldosterone and PRA were correlated with diastolic pressure in those with hypertension $(r=0.680 ; p=0.003$ and $r=0.608 ; p=0.01)$.

CONCLUSIONS: There is no direct correlation between aldosterone or SOD and Insulin resistance, and inflammation in men with central obesity. The correlation between PRA and MetS might be through insulin resistance mechanism. We found significant correlation between PRA, HDL-C and SOD. Increased aldosterone was ccorrelated with elevated triglycerides, thus possibly increasing the risk of MetS.

KEYWORDS: Central obesity, aldosterone, plasma renin activity, superoxide dismutase, inflammation, insulin resistance.

Indones Biomed J $2011 ; 3$ (2) : 116-121 


\section{Introduction}

The high prevalence of obesity is known to affect 50 to $60 \%$ of a nation's population. Obesity contributes to hypertension, high serum cholesterol, low HDL-C and hyperglycaemia, therefore it is associated with insulin resistance, metabolic syndrome (MetS), and higher cardiovascular disease (CVD) risks. The risk of serious health consequences in type 2 diabetes, chronic heart disease and a range of other conditions, including cancer, have been shown to rise with an increase in body mass index (BMI). Obviously central obesity is more accurate and reliable measure of MetS than BMI $(1,2)$.

The International Obesity Task Force reported that 1.7 billion of the world's population was already at a heightened risk of weight-related, non-communicable diseases such as type 2 diabetes. The mechanism by which excessive body fat causes insulin resistance and impairs glucose metabolism is not clearly defined. But fat stores (particularly visceral adipose tissue) are an important cause of increased free fatty acid and triglyceride in the skeletal muscle, which impairs insulin secretion, thus raising blood glucose levels and the likelihood of developing diabetes. Excess visceral adipose tissue also releases inflammatory cytokines that increase insulin resistance in skeletal muscles $(1,2)$. The most commonly used inflammatory marker is high-sensitivity CRP (C-reactive Protein).

$\mathrm{C}$-reactive protein (CRP) is an acute phase reactant used as a marker of inflammation. Mild elevations of CRP levels even when within the clinically 'normal' range can independently be predictive of future cardiovascular events. The causes for increased CRP in apparently healthy individuals is not really clear. Recently, elevated CRP level has been known to be associated with the features of insulin resistance syndrome, abdominal obesity, high fasting glucose, hyperinsulinaemia and insulin sensitivity, high triglyceride and low HDL-C. Visceral adipose tissue measures appear to be more closely linked with MetS and can be an important source for pro-inflammatory cytokine production in healthy individuals (3).

Obvioulsy, renin angiotensin aldosterone system (RAAS) plays a crucial role in blood pressure (BP) control as well as in the progression of CVD and renal disease. (4) The components of renin-angiotensin (RA) system are fully represented in the adipose tissue and are upregulated in obesity due to enhanced circulating angiotensinogen levels. The local RA system plays a role in adipocyte differentiation and possibly in body-fat accumulation. In humans, angiotensin II produced by mature adipocytes appears to inhibit differentiation of adipocyte precursors, thus decreasing the percentage of small insulin-sensitive adipocytes (5).

Additionally, plasma aldosterone, but not Plasma Renin Activity (PRA), is associated with the MetS per $s e$, independent of the association with its separate components. Differential association of PRA and aldosterone with other components of MetS suggest different underlying mechanisms in MetS (6).

Although the role of renin and aldosterone in BP regulation is well known, less is known about association of renin and aldosterone with MetS. Several observations suggest that plasma aldosterone plays a role in BP regulation in obesity. In addition, several studies have reported an association between aldosterone and/or PRA and blood lipid and insulin restance, which are components of MetS $(7,8)$. Heightened expression of RAAS eventually leads to increased reactive oxygen species (ROS).

Increased ROS production in accumulated fat contributes to MetS. It causes increased nicotinamide adenine dinucleotide phosphate (NADPH) oxidase and decreased antioxidant enzymes. Increased ROS production from accumulated fat also leads to increased oxidative stress in blood, hazardously affecting other organs including the liver, skeletal muscle, and aorta (9).

Adefense mechanism in order to eliminate the damage caused by ROS is developed through enzymatic convertion into its less toxic compound. Such enzymes are generally referred to as antioxidant enzymes, including Superoxide Dismutase (SOD), catalase, glutathione peroxidase, and glutathione s-transferase. SOD reduces superoxide ion into peroxide ion. Some researches showed that decreased SOD would increased the risks of vascular diseases (10).

Recently SOD was known to be related with insulin resistance, probably by increasing half-life of endothelial nitric oxide synthase (eNOS), thus reducing the risk of insulin resistance (10). Some studies have shown that an activated RAAS was associated with cardiovascular risk factor clustering, but unfortunately no studies have been done as yet on association of RAAS with insulin resistance and inflammation. The aim of this study was to assess the association between plasma Aldosterone, PRA and SOD and inflammation (hsCRP), and insulin resistance (HOMA-IR) in central obesity. 


\section{Methods}

This cross-sectional study recruited 80 male subjects with central obesity who were divided into 2 groups, namely the group of subjects who fulfilled the MetS criteria and the other group of those who did not. We collected serum samples from the 80 centrally obese men, aged 30-60 years, with waist circumference $\geq 90 \mathrm{~cm}$ (International Diabetes Federation criteria).

After an overnight fasting (10-12 hr), we recorded subjects' height, weight, BMIs, waist circumference, BPs and measured their biochemical variables as follows: concentration of fasting glucose, HDL-C, triglyceride, hsCRP, insulin, aldosterone, PRA and SOD. Insulin resistance was assessed by HOMA-IR, calculated as fasting glucose (in mmol/L) times (x) fasting insulin (in $\mathrm{mIU} / \mathrm{L}$ ) and divided by (:) 22.5. Insulin Resistance was defined as HOMA-IR > 3.5 (12).

We divided the study subjects into 2 groups, MetS and non MetS, According to the new IDF definition, subjects with central obesity are defined as those having waist circumference $\geq 90$ for Asian men, plus any 2 of these 4 factors: triglyceride level $>150 \mathrm{mg} / \mathrm{dL}(1.7 \mathrm{mmol} / \mathrm{L})$ or specific treatment for this triglyceride abnormality; HDL-C $<40 \mathrm{mg} / \mathrm{dL}(0.9 \mathrm{mmol} / \mathrm{L})$, or specific treatment for this lipid abnormality; systolic $\mathrm{BP} \geq 130$ or diastolic $\mathrm{BP} \geq 85$ $\mathrm{mmHg}$ or treatment of previously diagnosed hypertension; raised fasting plasma glucose (FPG) $\geq 100 \mathrm{mg} / \mathrm{dL}$ (5.6 $\mathrm{mmol} / \mathrm{L}$ ) or previously diagnosed type 2 diabetes.

Aldosterone (in $\mathrm{ng} / \mathrm{dL}$ ) was measured by radioimmunoassay method using Coat-A-Count ${ }^{\circledR}$ kit (Diagnostic Product Corporation, Los Angeles, CA, USA). PRA (in $\mathrm{ng} / \mathrm{mL} / \mathrm{hr}$ ) was assayed based on generated angiotensin 1 by radioimmunoassay method using Gamma Coat ${ }^{\circledR}$ Plasma Renin Activity 125RIA Kit (Diasorin, Still Water, MN, USA). Activity of SOD (in U/g Hb) was determined from whole blood using RANSOD reagent (RANDOX laboratories Ltd., Crumlin, UK).

Serum glucose, HDL-C, and triglyceride were determined using Prodia Clinical Laboratory routine procedures by Modular P800. Insulin was measured by immunochemilluminescent method using Immulite 2000. Serum hsCRP was measured by sensitive immunoassay technique (Diagnostic Product Corporation) using Immulite 2000.

\section{Statistical Analysis}

Statistical analysis were conducted using SPSS 11.5 software (SPSS Inc., Chicago IL, USA). Distributions of continuous variables were assessed for normality using Kolmogorov-Smirnov and for variance homogeneity using Leven's test. Independent t-tes was used to confirm the difference between variables in MetS group and those without MetS. Univariate-bivariate analysis, Pearson or Spearman's rho correlation analysis were performed to analyze the correlation between various components and biochemical measures. The results are presented in both narration and tables and graphs, $\mathrm{p}$ values $<0.05$ are considered as statistically significant.

\section{Results}

The subjects' anthropometric measurement (height, weight, BMI, waist circumference, and BP) and biochemical measurements (fasting glucose, fasting insulin, HDL-C, triglycerides, SOD, hsCRP, PRA, aldosterone) were shown in Table 1 . The results showed 29 subjects with MetS and 51 subjetcs were without MetS.

Table 1 shows that there were no significant differences in age, waist circumference, BMI, systolic $\mathrm{BP}$, diastolic BP, aldosterone, PRA, and SOD between MetS and non MetS groups. But there were significant differences in fasting glucose, HDL-C, triglyceride, and HOMA-IR between MetS and non MetS groups.

Unpaired t-test was performed to compare means of waist circumference, aldosterone, PRA, SOD, hsCRP between MetS and non MetS. The mean of PRA was not significantly higher in non MetS group than in the MetS group $(2.54 \pm 2.10$ vs $2.29 \pm 1.85 \mathrm{ng} / \mathrm{mL} / \mathrm{hr})$. Mean aldosterone was not significantly higher in MetS group than in the non MetS group $(5.29 \pm 3.33 \mathrm{ng} / \mathrm{dL}$ vs 5.88 $\pm 3.81)$. In addition HOMA-IR value were not normally distributed, so we transformed them into logarithmic (table 2). Mean HOMA-IR in the MetS group was significantly higher than that in the non MetS group $(2.87 \pm 3.10$ vs $1.69 \pm 0.88) \mathrm{p}<0.05$.

\section{Correlation of Aldosterone, PRA, SOD, hsCRP and HOMA-IR with MetS components in centrally obese men}

Pearson or Spearman's correlations test was performed to assess correlation of Aldosterone, PRA, SOD, hsCRP and HOMA-IR with MetS components.

The result showed there were no correlations of aldosterone, PRA, and SOD with inflammation (hsCRP) and insulin resistance (HOMA-IR) in central obese men. (Table 2). 
Table 1. Clinical and Biochemistry characteristic of subjects

\begin{tabular}{|c|c|c|c|c|}
\hline \multirow[t]{2}{*}{ Parameter } & \multicolumn{3}{|c|}{ MEAN } & \multirow[b]{2}{*}{ p } \\
\hline & Obese & Non MetS & MetS & \\
\hline $\mathrm{N}$ & 80 & 51 & 29 & - \\
\hline Age (years) & $40.70 \pm 8.20$ & $40.70 \pm 7.70$ & $40.90 \pm 9.10$ & 0.913 \\
\hline Waist Circumference $(\mathrm{cm})$ & $99.28 \pm 7.83$ & $98.78 \pm 7.42$ & $100.17 \pm 8.57$ & 0.450 \\
\hline Systolic BP (mmHg) & $115.40 \pm 10.50$ & $113.50 \pm 9.40$ & $118.80 \pm 11.60$ & 0.310 \\
\hline Diastolic BP (mmHg) & $79.10 \pm 8.02$ & $77.60 \pm 6.70$ & $81.70 \pm 9.40$ & 0.280 \\
\hline Weight (kg) & $80.60 \pm 10.90$ & $79.90 \pm 9.80$ & $81.90 \pm 12.80$ & 0.453 \\
\hline BMI $\left(\mathrm{kg} / \mathrm{m}^{2}\right)$ & $28.66 \pm 3.53$ & $28.53 \pm 2.97$ & $28.90 \pm 4.40$ & 0.663 \\
\hline Fasting Glucose (mg/dL) & $88.62 \pm 11.58$ & $86.58 \pm 8.26$ & $92.20 \pm 15.35$ & 0.036 \\
\hline HDL-C (mg/dL) & $42.47 \pm 7.82$ & $45.72 \pm 7.62$ & $36.75 \pm 3.99$ & $<0.0001$ \\
\hline Triglyceride (mg/dL) & $165.38 \pm 82.40$ & $132.49 \pm 49.10$ & $223.24 \pm 96.97$ & $<0.0001$ \\
\hline $\mathrm{SOD}(\mathrm{U} / \mathrm{gHb})$ & $1293.40 \pm 175.30$ & $1296.80 \pm 185.90$ & $1287.40 \pm 157.70$ & 0.819 \\
\hline $\operatorname{hsCRP}(\mathrm{mg} / \mathrm{L})$ & $2.28 \pm 1.54$ & $2.18 \pm 1.57$ & $2.45 \pm 1.48$ & 0.449 \\
\hline Fasting Insulin ( $\mu \mathrm{lU} / \mathrm{mL})$ & $9.22 \pm 6.99$ & $7.83 \pm 3.81$ & $11.69 \pm 10.12$ & 0.017 \\
\hline HOMA-IR & $2.12 \pm 2.06$ & $1.69 \pm 0.88$ & $2.88 \pm 3.11$ & 0.013 \\
\hline PRA (ng/mL/hr) & $2.45 \pm 2.01$ & $2.54 \pm 2.10$ & $2.29 \pm 1.85$ & 0.559 \\
\hline Aldosterone (ng/dL) & $5.51 \pm 3.50$ & $5.29 \pm 3.33$ & $5.88 \pm 3.81$ & 0.471 \\
\hline
\end{tabular}

HDL-C High Density Lipoprotein Cholesterol ; SOD Superoxide Dismutase ; hsCRP High sentitive CReactive Protein; HOMA IR Homeostatis Model Assesment of Insulin Resistance ; PRA Plasma Renin Activity.

Table 2. Correlation between Aldosterone, PRA, SOD, hsCRP, and HOMA-IR with each component of MetS in men with central obesity

\begin{tabular}{lccccc}
\hline & $\begin{array}{c}\text { Aldosterone } \\
\text { Parameter }\end{array}$ & $\begin{array}{c}\text { PRA } \\
\text { r }\end{array}$ & $\begin{array}{c}\text { SOD } \\
\mathbf{r}\end{array}$ & $\begin{array}{c}\text { hsCRP } \\
\mathbf{r}\end{array}$ & $\begin{array}{c}\text { Log HOMA } \\
\mathbf{r}\end{array}$ \\
\hline PRA (ng/mL/hr) & $0.389^{* *}$ & 1 & $0.443^{*}$ & -0.95 & 0.08 \\
Aldosterone (ng/dL) & 1 & $0.389^{*}$ & -0.121 & 0.040 & 0.034 \\
Log HOMA & 0.034 & 0.168 & 0.176 & $0.303^{* *}$ & 1 \\
Waist Circumference(cm) & 0.014 & -0.118 & 0.08 & $0.437^{*}$ & $0.374^{* *}$ \\
Sistolic BP (mmHg) & -0.047 & -0.133 & 0.119 & -0.123 & 0.054 \\
Diastolic BP (mmHg) & -0.083 & -0.014 & 0.101 & -0.134 & 0.08 \\
HDL-C (mg/dL) & -0.165 & $0.273^{*}$ & 0.510 & -0.167 & -0.150 \\
Fasting Glucose (mg/dL) & -0.173 & 0.033 & 0.153 & -0.021 & $0.627^{* *}$ \\
Triglyceride (mg/dL) & $0.234^{* *}$ & 0.037 & 0.330 & -0.110 & $0.287^{* *}$ \\
SOD (U/gHb) & -0.121 & $0.220^{*}$ & 1 & -0.220 & 0.176 \\
hsCRP (mg/L) & 0.040 & 0.044 & -0.022 & 1 & $0.303^{* *}$ \\
BMI (kg/m²) & -0.060 & -0.121 & -0.62 & $0.387^{*}$ & $0.382^{* *}$ \\
\hline
\end{tabular}

PRA Plasma Renin Activity; HOMA IR Homeostatis Model Assesment of Insulin Resistance; BP Blood Pressure ; HDL-C High Density Lipoprotein Cholesterol ; S OD Superoxide Dismutase ; hsCRP High sentitive C Reactive Protein.

Significant correlation : ${ }^{\star} p<0.05 \quad{ }^{* \star} p<0.001$ 


\section{Discussion}

The main finding of this study showed that there were no direct correlation of aldosterone and PRA with inflammation (hsCRP) and insulin resistance (HOMAIR) in centrally obese men. Also there was no correlation between SOD and inflammation (hsCRP), or insulin resistance (HOMA-IR). The data showed that the average PRA and SOD in MetS group was lower than those in the non MetS group.

Aldosterone level was higher in the MetS group than in the non MetS group. Inflammation was considered to occur initially in obese subjects before they met the MetS criteria; this inference was derived form the correlation data.

We found no significant correlation between aldosterone and hsCRP, HOMA-IR or SOD in this study. But surprisingly, there were significant correlation between aldosterone and PRA $(r=0.389)$ and triglyceride $(r=0.234)$, suggesting that there was an association between aldosterone and one of MetS component, namely triglyceride. The blockage of the RAAS may increase the number of small, newly differentiated adipocytes, which are known to be more insulin sensitive than older, larger adypocytes. This outlook may provide a novel explanation for the unexpected recent findings that suggested that RAAS blockade may lower the risk of type 2 diabetes (11). An impaired ability to differentiate new fat cells, possibly manisfested as enlargement of existing ones, may in part play a role in the development of type 2 diabetes. The mechanisms may comprise promoting the storage of excess lipids in other organs like liver, muscle or pancreas (lipotoxicity hypothesis), so it may cause distraction of lipid metabolism (11).

The results of our study showed that there were no correlation between PRA and inflammation or insulin resistance. But in sub-group analysis of MetS and non MetS, we found a positive correlation between PRA and $\log$ HOMA-IR $(r=0.471, p=0.010)$ in MetS group.

Mature adipocytes express all component of RAAS, including angiotensinogen (AGT), the sole precursor of Ang II, ACE, and Chymase. RAAS activity would be expressed in accumulated fat. RAAS blockage would increase small newly differentiated adiposity, which are more sensitive to insulin. Thus highly expressed RAAS would decrease small newly - insulin sensitive - adipocyte, then this would increase the risk of insulin resistance (11).
We found a positive correlation between PRA and HDL-C in MetS group $(r=0.493 \mathrm{p}<0.01)$. However, Alikmets et al., (13) reported a negative correlation between PRA and HDL-C. This difference might be due to different study methods used in the two studies. Alikmets et al. studied the subjects in the essential hypertensive group, but in this study we didn't divide them into normotensive and hypertensive groups, so difference in both studies' findings might also be due to the different subjects' positions during samples collection.

In our study collection of blood samples from the study subjects was done in a sitting position. The instruction reference to the reagent kit stated it should be performed only in standing or lying position. Another study performed on 15 healthy individuals has shown that there were no significant changes occured in PRA concentration in lying position or sitting position. Therefore this fact should be one of our consideration.

The level of PRA is also influenced by natrium intake, position during blood sampling, sampling time, hypertension medication and capthesin D (14). As conversion of Angiotensinogen into angiotensin-1 is influenced by capthesin D (14), measurement of capthesin D should be included in further studies.

\section{Conclusion}

There was no direct correlation between Aldosterone or SOD and Insulin resistance, and inflammation. The correlation between PRA and MetS might be through insulin resistance mechanism. We found a significant correlation between PRA, HDL-C and SOD. Increased Aldosterone was correlated with elevated Triglycerides, thus possibly increasing the risk of MetS.

\section{Acknowledgements:}

The authors are grateful to the Prodia Education and Research Institute for the invaluable support.

\section{References:}

1. International Diabetes Federation. The IDF Consensus Worldwide Definition of the Metabolic Syndrome. 2005 (Online). (www.IDF.org.cited 12 August 2007).

2. International Diabetes Federation. Metabolic Syndrome-Driving the CVD Epidemic. 2005 (Online). (www. Idf.org. cited 12 August 2007). 
3. Forouhi NG, Sattar N, McKeigue PM. Relation of C-Reactive Protein to Body Fat Distribution and Features of the Metabolic Syndrome in Europeans and South Asians. Int J Obes Relat Metab Disord 2001; 25: 1327-31.

4. Laragh J. Laragh's Lessons in Pathophysiology and Clinical Pearls for Treating Hypertension. Am J Hypertens. 2001; 14: 307-10.

5. Strazullo P, Galleti F. Impact of Renin-Angiotensin System on Lipid and Carbohydrate Metabolism. Curr Opin Nephrol Hypertens. 2004; 13: 325-32.

6. Bochud M, Nussberger J, Bovet P. Plasma Aldosteron is Independently Associated with the Metabolic Syndrome. Hypertension. 2006; 48: 239-45.

7. Goodfriend TL, Kelley DE, Goodpaster BH, and Winters SJ. Visceral Obesity and Insulin Resistance are Associated with Plasma Aldosterone Levels in Women. J Obes Res. 1999; 7: 355-62.

8. Goodfriend TL, Egan BM, Kelley DE. PlasmaAldosteron, Plasma Lipoprotein, Obesity, and Insulin Resistance in Humans. Prostaglandins Leukot Essent Fatty Acids. 1999; 60: 40105 .

9. Furukawa S, Fujita T, Shimabukuro M, Iwaki M, Yamada $Y$,
Nakajima Y, et al. Increased Oxidative Stress in Obesity and Its Impact On Metabolic Syndrome. J Clin Invest. 2004; 12 : $1752-61$.

10. Undurti, N. Is Metabolic Syndrome X an Inflammatory Condition?. Exp Biol Med. 2002; 227: 989-97.

11. Janke J, Engeli S, and Gorzelniak K. Mature Adipocytes Inhibit in Vitro Dinfferentiation of Human Preadipocytes via Angiotensin Type 1 Receptors. Diabetes. 2002; 51: 1699707.

12. Stern SE, Williams $K$, Ferrannini E, DeFronzo RA, Bogardus $C$, Stern MP. Identification of Individuals with Insulin Resistance Using Routine Clinical Measurements. Diabetes. 2005; 54: 333-9.

13. Allikmets K, Parik T, Teesalu R. Association between Plasma Renin Activity and Metabolic Cardiovascular Risk Factors in Essential Hypertension. J Internal Medicine. 1996; 239: 49-55.

14. Naseem HR, Hedegard W, Henry TD, Lessard J, Sutter $K$, and Katz SA. Plasma Cathepsin D Isoforms and Their Active Metabolites Increase after Myocardial Infarction and Contribute to Plasma Renin Activity. Basic Research in Cardiology. 2005; 100: 139-46. 